Department of Epidemiology and Virus Laboratory, School of Public Health, University of Michigan, Ann Arbor, Michigan

\title{
Initial Stages of the Interaction of HeLa Cells with Poliovirus*
}

\author{
$\mathrm{By}$ \\ Francis E. Payne **, Hilda Kurtz, and W. Wilbur Ackermann \\ With 7 Figures \\ (Received June 21, 1957)
}

As a consequence of the usual interaction of viruses and cells, one detects an initial loss of viral activity, a viral increase and destruction of cells. In such cases the reaction may be followed in terms of any of the resultant effects. However, all interactions may not be productive of virus; nor result in cellular destruction and viral activity may lend itself to competing reactions. To follow the reaction by a single effect may preclude the observation of unexpected phenomena. The present study is centered upon the eytopathogenic effect of the poliovirus and for this reason its reaction with the HeLa cell is followed primarily in terms of cellular destruction. For this purpose a method has been developed for quantitating cellular injury. By the application of the method, the kinetics of two early phases of the interaction of virus and cell have been investigated.

\section{Methods and Materials}

Virus. Two lines of the Mahoney strain of type 1 poliovirus were used. These stock cultures were not purified by clonal isolation, however, many of their characteristics remain fixed when passaged in a standard manner. One line was adapted to HeLa cells by 19 serial passages in these cells. When plated upon monolayers of HeLa cells, uniformly large plaques with regular edges are formed and the number of plaques is

* This work was supported by a grant from the National Foundation for Infantile Paralysis.

** Dr. F. E. Payne is a Fellow of the National Foundation for Infantile Paralysis. 
maximal by the third day. The second line (used in only one experiment described here) has not been passaged in HeLa cells but was maintained in monkeys and recently in tissue cultures of monkey kidney cells. This line when plated upon HeLa cells produces small, irregular plaques which increase in number for several days. Both lines are neutralized by immune serum prepared in monkeys with the prototype 1, Brunhilde strain, poliovirus'.

Host Cells. The line of HeLa cells used in all experiments and in monolayers for plaque titration was obtained originally from Dr. J. T. Syverton. The lines used in these experiments have not been deliberately subjected to selection as by using clones derived from single cells.

Growth Medium. The cells were grown in human serum (40\%) and balanced salt $(60 \%)$. Those cultures prepared for plaque titrations were cultivated in human serum $(20 \%)$ and Eagle's basal medium $(80 \%)^{2}$. All experimental cultures contain as routine penicillin, streptomycin and mycostatin; however, stock cultures are maintained without antibiotics.

Maintenance Medium. For the propagation of virus or maintenance of cultures for a short period free of human antibodies, equine serum $(10 \%)$ and Scherer's maintenance solution $(90 \%)$ have been ised ${ }^{3}$.

Antisera. Type specific hyperimmune monkey sera were prepared using active poliovirus (type 1, Mahoney strain) which had been propagated in HeLa cells. One milliliter of undiluted tissue culture fluid emulsified in an equal-amount of Bayol-F (Eśso Standard Oil Co., Philadelphia) containing 10\% Arlacel-C (Atlas Powder Co., Wilmíngton, Delaware) was inoculated intramuscularly at bi-weekly intervals for an initial series of three injections. Subsequent vaccinations were at intervals of four or more weeks and the monkeys were bled two weeks after each booster-injection. Serum from individual animals was stored at $4^{\circ} \mathrm{C}$. Serum prepared in this manner and tested in stationary tube cultures of HeLa cells may neutralize 100 TCID $_{50}$ of virus at dilutions from $1: 3200$ to $1: 6400$.

Viral Titrations. The plaque assay method of Dulbecco and Vogt ${ }^{4}$ was modified to allow the use of HeLa cell cultures. Two ounce prescription bottles inoculated with 750000 trypsin-suspended cells in $4 \mathrm{ml}$ of Eagle's basal medium ${ }^{2}$ containing $20 \%$ adult human serum were incubated at $37^{\circ} \mathrm{C}$ until a continuous cell layer was formed: The growth medium was changed every other day.

Each culture was washed three times with maintenance medrtim to remove any residual human serum of the growth medium. After being rinsed with $2 \mathrm{ml}$ of medium the culture was incubated for two to three hours in $3 \mathrm{ml}$ of fresh fluid. This was then replaced with $2 \mathrm{ml}$ of maintenance medium which was removed just prior to adding the virus. 
A volume of $0.5 \mathrm{ml}$ of virus suspension in balanced salt solution at the appropriate dilution was pipetted onto the cell layer of each of four bottles. After two hours incubation at $37^{\circ} \mathrm{C}$, the infected cultures were overlayed with $4 \mathrm{ml}$ of melted (at $43^{\circ} \mathrm{C}$ ) agar mixture $(1.5 \%$ Noble agar; Difco, in maintenance solution containing $10 \%$ equine serum and $0.002 \%$ neutral red). After the agar had solidified at room temperature the bottles were inverted for incubation at $37^{\circ} \mathrm{C}$.

Each day, from the second to the fifth day following infection, the number of newly appearing plaques was added to the previous day's count. Titers were expressed as PFU (plaque forming units) per milliliter and were calculated from the total number of plaques that had appeared by the fifth day.

\section{Experimental and Results}

Cytopathogenic Effects of Infected Tissue Culture Fluids. The cytopathogenic effect upon HeLa cells of a tissue culture fluid infected with poliovirus was followed. The manifestation of cytopathology measured was the inability of the injured cells to remain attached to the glass substrate. The cells which did remain were counted at various times following a brief exposure of the culture to the infected fluid.

Several replicate bottle-cultures of HeLa cells which contained approximately $7 \times 10^{6}$ cells in a monolayer attached to one interior surface were selected. The cultures were washed to remove traces of human serum and then were exposed to $4 \mathrm{ml}$ of balanced salt solution containing $0.3 \mathrm{ml}$ of undiluted tissue culture fluid. Thus $53 \times 10^{6} \mathrm{PFU}$ (plaque forming units) of virus were employed. After 15 minutes, the cells were washed five times with $5 \mathrm{ml}$ portions of balanced salt solution and incubated further at $37^{\circ} \mathrm{C}$ with $8 \mathrm{ml}$ of maintenance solution containing $10 \%$ equine serum. Cultures not counted by 105 minutes after exposure to virus were supplemented at that time with $0.5 \mathrm{ml}$ of immune monkey serum containing 600 units of poliovirus antibody. (The addition of serum at this time does not interfere with the effects of the inoculum but is present to prevent secondary effects of the viral progeny which will begin to appear at three to four hours.)

At various times, from one to 48 hours after exposure, cultures were examined and the degree of cytopathology quantitated. To do this, the cultures were gently washed with calcium free balanced salt solution to remove destroyed cells. Further treatment with $0.02 \%$ "Versene". (tetra sodium ethyline diamine tetra acetate) in calcium free balanced salt solution yielded a suspension of healthy cells which had remained attached to the glass. There is some evidence that remaining injured cells are not preserved in the presence of "Versene". The number of 
cells in the suspension was then determined in a hemocytometer. Control cultures not exposed to infected fluids were carried through the procedure and their cells were counted at one, three or 24 hours.

These control cultures did not vary significantly in cell count. Hence, in Fig. 1 where the data are a composite of two comparable experiments, the counts of the control cultures are expressed as 100 and the experimental ones as percentages of the control. The data show that cell losses occur within two intervals, one within the first hour and the second between the sixth and twelfth hour.

Rate of Development of Cytopathology

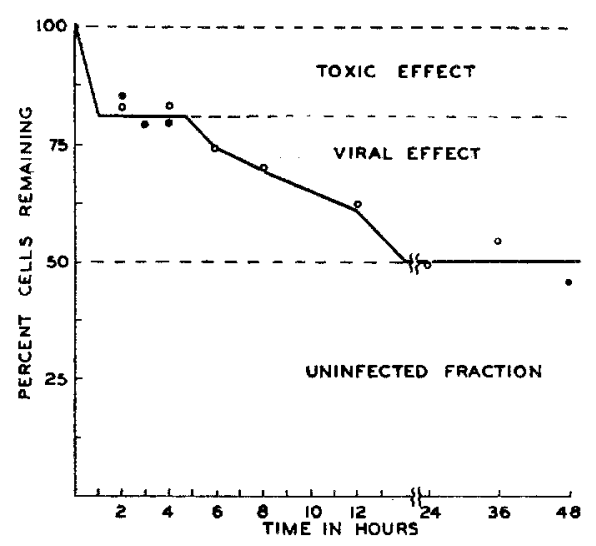

Fig. 1

Data are a composite of two experiments. Each point represents a single experimental culture. \% cells remaining $=100 \times$ cell count of experimental culture/cell count of control culture.

Each experimental culture was exposed to $7.4 \mathrm{PFU}$ of virus/cell for 15 minutes.

Between the first and fourth hour and in the period from 24 to 48 hours the cell count is constant. The infected tissue culture fluid seems to produce two distinct cytopathogenic effects, one very rapid and early, and a second which follows the viral latent period and begins at approximately the time the release of virus from cells is known to occur. While the exact duration of the second period of cytopathogenic effect has not been determined, it most likely terminates shortly following the twelfth hour. From these data, it is clear that successive secondary effects do not occur when immune serum is present in sufficient concentration. Further, in the cultures retained for 24 hours or longer, the extra-cellular fluids were found to be non-infectious when removed and tested, without dilution, in other cultures. Any virus which may have been produced as a result of infection was neutralized and incapable of producing further detectable cytopathology in the culture of its origin. As will be shown in the successive sections, the serum in competition with cells for virus produced secondarily has an advantage in that the serum can yet react with virus for some time after attachment.

Influence of Immune Serum. Upon Cytopathogenic Effect. Three replicate bottle cultures of HeLa cells were selected which contained approximately $7.5 \times 16^{6}$ cells. These were prepared for infection as described in the previous section. Two of these were inoculated with 
$20 \times 10^{6} \mathrm{PFU}$ of poliovirus. The three cultures were incubated 30 minutes at $37^{\circ} \mathrm{C}$ and then washed five times with $5 \mathrm{ml}$ portions of balanced salt solution. At once, maintenance solution containing 600 units of type 1 poliovirus antiserum was added to one of the infected cultures. The remaining ones received only maintenance solution until three hours later when they were supplemented with 600 units of immune serum. At the end of 24 hours all cultures were examined and the degree of cytopathology quantitated by the procedure described in the previous section.

From a comparison with the uninfected control, it was calculated that $73 \%$ of the cells had survived in the culture treated with serum immediately after removal of virus. In the second infected culture incubated three hours after removal of virus and before the addition of serum only $47 \%$ of the cells survived.

Since the immune serum in each case was added before the viral productive period of the sequence began and was present throughout this period in sufficient quantity to neutralize any virus which may have been produced, the cellular destruction is regarded as precipitated by the action of the viral inoculum. In each case the period of exposure to the inoculum was the same and the free virus was removed before the addition of serum. The high efficiency of the washing procedure was verified by plating the virus obtained in each wash fluid. Hence, any effects of serum must be upon a cell-virus complex and the differential in the effect of addition of serum at the two times implies that the ability of serum to act upon the complex is conditioned by the age of the complex.

The interpretation is that at 30 minutes at least $53 \%$ of the culture had reacted; $27 \%$ was in a state resistant to the action of serum and $26 \%$ was in a sensitive state. The cells refractory to immune serum include those reacting to the toxic effect of the infected fluid. During

Table I

\begin{tabular}{c|c|c}
\hline \multicolumn{2}{|c|}{ Time (Minutes) } & $\begin{array}{c}\text { Percent of Cells } \\
\text { Destroyed at 24 hours }\end{array}$ \\
\hline Period of Exposure to Virus * & $\begin{array}{c}\text { Point of Addition of } \\
\text { Antiviral Serum }\end{array}$ & \\
\hline $0-30$ & 30 & 27 \\
$0-30$ & 180 & 53 \\
Control (no virus) & 180 & 0
\end{tabular}

Therefore, at 30 minutes $26 \%, 53 \%$ minus $27 \%$, of the cells were complexed with virus in such a way that they were protected by the addition of immune serum.

* Each culture was inoculated with approximately $2.7 \mathrm{PFU} / \mathrm{cell}$. 
some part of the interval from 30 minutes to 210 minutes, the sensitive infected cells $(26 \%)$ developed resistance to serum action (Table I).

Once it is possible to determine the fraction of cells infected at a particular time and the portion thereof sensitive to the action of serum, it becomes feasible by the addition of serum at suitable intervals to

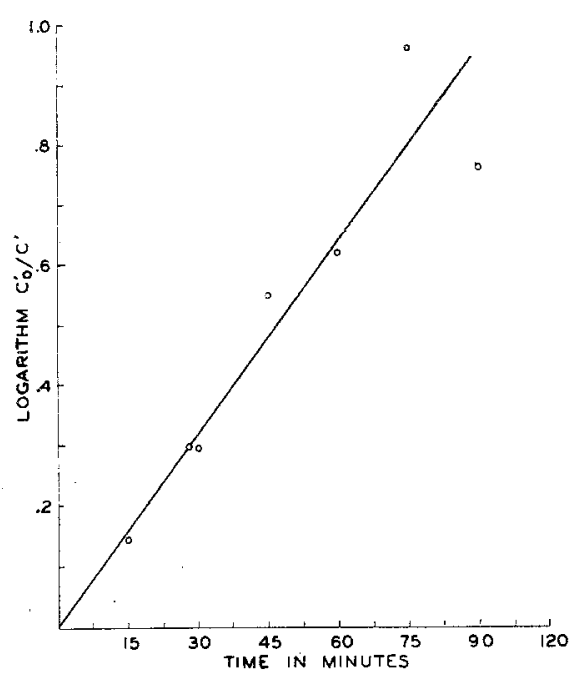

Fig. 2. Rate of Development of Resistance to Immune Serum: Reaction II

The fraction of cells sensitive to the action of antiviral serum at the end of the period of exposure to virus,

$$
C_{0}^{\prime}=C_{30}-C_{180}
$$

and the fraction sensitive to serum at any time $t$, designated $O^{\prime}$ is $C_{t}^{\prime}=O_{t}-O_{180}$ where $C_{180}=$ the fraction of cells surviving after 30 minutes exposure to virus and 150 minutes incubation before addition of antiviral serum and $C_{30}=$ the fraction of cells surviving in cultures exposed to virus for 30 minutes and immediately supplemented with antiviral serum. Each point represents an average of values obtained from two cultures.

Each culture was exposed to 3.3 PFU of virus/cell for 30 minutes, washed, and 600 units of antibody added at appropriate intervals. measure the rate at which serum resistance develops and the limit in time when the early effect of serum approaches a minimum. The measurement of the rate of this reaction, which will be designated as Reaction II, is illustrated in the following experiment.

Rate of Development of Serum Resistance. Seventeen replicate bottle cultures of HeLa cells were prepared for infection and. sixteen were inoculated with poliovirus as described in the preceding section. After 30 minutes of exposure to virus $\left(16 \times 10^{6} \mathrm{PFU}\right.$ in $\left.8 \mathrm{ml}\right)$, all cultures were washed five times with balanced salt solution. At each 15 minute interval from 0 to 180 minutes after removal of the virus, an addition of immune serum was made to two of the infected cultures to provide duplicate determinations. Following a further incubation period of 24 hours, the surviving cells were counted.

From the control culture not infected, and the cultures receiving immune serum at intervals after infection, the fraction resistant to serum at each time interval was determined. Progressively with time this fraction was found to increase until by 90 minutes there was a pronounced flattening of the curve to produce a plateau. The addition of serum at times after 90 minutes permitted only a slightly greater degree of cellular destruction. 
If one assumes the fraction of cells destroyed by 24 hours (expressed as the difference of surviving cells, $C_{0}-C_{180}$ ) in the culture receiving serum at 180 minutes approximates the total number - of cells infected during the period of exposure to virus, then the difference between this fraction and that destroyed following the addition of serum at 30 minutes $\left(C_{0}-C_{30}\right)$ is the fraction infected but sensitive to serum at the beginning of the study period, i. e., 30 minutes. When this is designated as $C_{0}{ }^{\prime}$ :

$$
\mathrm{C}_{0}{ }^{\prime}=C_{30}-C_{180}
$$

and the infected fraction sensitive to serum at any time $t$, designated $C^{\prime}$, obtains from $C_{t}-C_{180}$.

In Fig. 2, the logarithm of $C_{0}^{\prime} / C^{\prime}$ is plotted as a function of time. It will be noted that the data fall about a straight line which passes through the origin. The function can be expressed as:

$$
\log C_{0}^{\prime} / C^{\prime}=k t \text {. }
$$

Thus, the reaction proceeds at a rate determined by the instantaneous concentration of infected sensitive cells, indicating first order kinetics. The data are least reliable at the late time intervals where small changes are measured against a relatively large background of uninfected cells.

It is apparent that if one varies the period of exposure of cells to virus, allows the resistance to serum to develop in normal medium, and allows cytopathology and virus production to proceed in the presence of immune serum, then the rate at which the initial cell-virus complex (which is stable to dilution) is formed can be followed. The technique for measuring the rate of this reaction, which will be designated as Reaction $I$, is illustrated in the following experiment.

Rate of Formation of the First Stable Cell-virus Complex. Nine replicate cultures of HeLa cells were selected and prepared for infection. Eight of these received $96 \times 10^{6} \mathrm{PFU}$ of poliovirus and one was retained as a control. The cultures were incubated at $37^{\circ} \mathrm{C}$ and at various times in the interval from 0 to 120 minutes, one infected culture was removed, washed five times to remove the virus inoculum and incubated further with maintenance medium. At 210 minutes after the original addition of virus and 90 minutes after the free virus was removed from the last culture, immune serum was added to all cultures and they were then ineubated at $37^{\circ} \mathrm{C}$ for 24 hours. Thus cultures of cells were obtained which had been exposed to virus for $6,15,30,45,60,75,90$ or 120 minutes. After removal of free virus, sufficient time had been allowed for Reaction II to be completed and then serum was added to prevent secondary spread of infection. At 24 hours the cultures were washed, treated with "Versene" to prepare a suspension of the remaining normal cells, $C$, which were then counted. 
From the count in the control bottle the initial number of cells, $C_{0}$, in the cultures was determined. In Fig. 3 the resulting data are presented. The logarithm of $C_{0} / C$ is plotted for each of the experimental times. It will be noted that there is a linear relationship between time and the logarithm of $C_{0} / C$. This indicates that the rate, at any time during the reaction, is a function of the number of susceptible cells remaining in the culture at that time. In this time range the reaction appears to be first order.

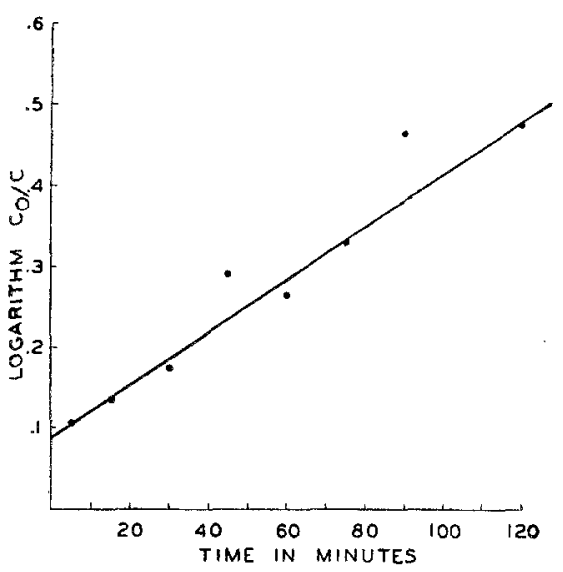

Fig. 3. Rate of Initiation of Infection: Reaction I Each point represents data obtained from a single culture exposed to 5.4 PFU of virus/cell for the indicated time interval. Antiviral serum was added to each culture 90 minutes after termination of the exposure period and the number of surviving cells, $C$, was determined at 24 hours. The initial number of cells, $C_{0}$, was obtained from the count of an uninfected control culture.

If $d C / d t$ were a function only of $C$, one would expect that the plot of $\log C_{0} / C$ against $t$ would yield a straight line passing through the origin. However, when fresh preparations of this line of poliovirus made in HeLa cell cultures were used as a source of virus, this was never seen. Extrapolation to zero time always produced an apparent intercept. This deviation indicates a cytopathogenic principle present in the viral inoculum which reacts with kinetics quite different from the usual virus action. If a rapid cytotoxic effect were produced, the estimate of $C_{0}$ from the control culture would be in error by some amount $x$, whose value might depend on the size of the inoculum,

i. e., the amount of toxin present. In this case one would expect that:

$$
\ln \left(C_{0}-x\right) / C=m t
$$

or by rearrangement:

$$
\ln \left(C_{0} / C\right)=m t+n
$$

where $n$ is substituted for $\ln \left(C_{0} /\left(C_{0}-x\right)\right)$ thus the plot of $\ln \left(C_{0} / C\right)$ against time would give a straight line function with an intercept on the ordinate whose magnitude depends upon the relative values of the original concentration of cells and the size of the inoculum.

The correctness of this interpretation of the present data is supported by that recorded in Fig. 1 where a cytotoxic principle was first suggested. It is supported further by the finding that another line of the Mahoney strain of type 1 poliovirus routinely passaged in monkey kidney culture upon transfer to HeLa cultures yielded viral preparations free of toxin 
(as measured in experiments of the type recorded in Fig. 1). Rate studies with this line of virus revealed a simple kinetic picture in which the plot of $\log C_{0} / C$ against time gives a straight line passing through the origin (see Fig. 4).

Under these conditions the concentration of virus does not appear to become limiting as the reaction proceeds and the rate of reaction is a function of the concentration of susceptible cells; however, the rate of reaction can be shown to be also a function of the virus concentration. This would follow if one were dealing with a pseudo-first order reaction in which case the slope, $m$, given above is the resultant of the viral concentration and a rate-reaction constant:

$$
\ln \left(C_{0} / C\right)=k V_{t}+n .
$$

To test this proposal, the relationship of the reaction rate to virus concentration was explored in the following type of experiment where the rate of Reaction I was determined using several concentrations of virus.

Relation of Rate Reaction Constant to Virus Concentration. Five groups of cultures of HeLa cells were exposed to different amounts of virus ranging from $9 \times 10^{6}$ to $48 \times 10^{6}$. In each group the periods of exposure to virus of individual cultures were 15,45 , or 75 minutes. Ninety minutes after the exposure to virus and subsequent

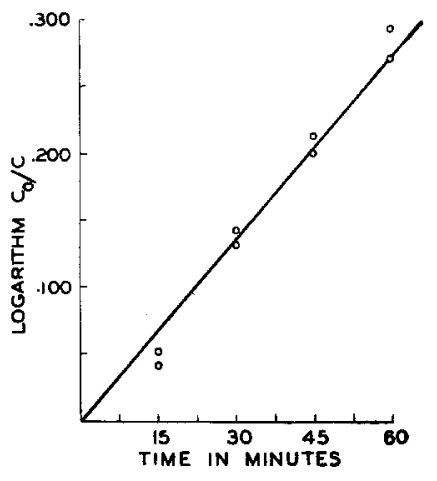

Fig. 4. Rate of Reaction I with Non-toxic Line of Poliovirus

The rate of Reaction $\mathbf{I}$ determined as in Fig. 3, but using a non-toxic line of poliovirus. At each interval duplicate cultures were studied and the results are plotted for both. Cultures were exposed to 5.2 PFU of virus/cell. washing of the cultures, immune serum was added and the cultures incubated further. Thus by the technique described in the previous section, the rate of Reaction I was determined in cultures of cells infected with five different concentrations of virus. The resulting data are plotted in Fig. 5 .

It should be noted that in other experiments which showed Reaction I to follow first order kinetics a multiplicity of exposure of five or more was employed. In the present experiment the exposure multiplicities were 5.6 and less.

Each point in Fig. 5 represents a single determination with a correspondingly limited accuracy, nevertheless straight lines were employed in examining the data. This was considered justifiable since the use of one concentration of virus in more detailed experiments did yield data which fall upon a straight line. These straight lines of 
differing slopes, all upon extrapolation to zero time intercept the ordinate. Both the intercept and the slopes are functions of the virus inoculum.

Apparently the reaction is a pseudo-first order reaction resulting from the use of an excess of virus and, when high multiplicities of exposure are employed, behaves as expressed by the above equation. The actual rates of reaction are determined by the rate reaction constant and virus

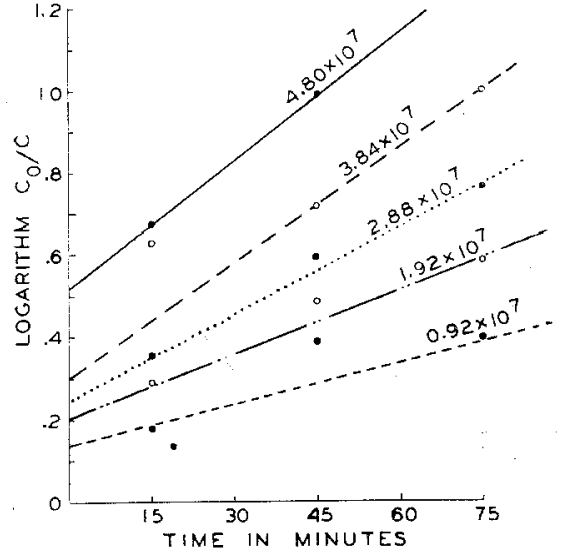

Eig. 5. Effect of Virus Concentration Upon Rate of Reaction 1

Five groups of replicate cuitures were exposed to different amounts of virus ranging from $0.92 \times 10^{7}$ to $4.80 \times 10^{7} \mathrm{PFU}$ as noted on individual ourves (multiplicities of exposure ranged from 1.12 to $5.6 \mathrm{PHU}$ (cell). In each group, periods of exposure to virus of individual cultures were 15,45 , and 75 minutes. Antiviral serum was added to each culture 90 minutes after termination of the exposure period and the number of surviving cells, $C$, was determined at $\mathbf{2 4}$ hours. The initial number of cells, $\mathbf{C}_{0}$, was obtained from the count of an uninfested control eulture. concentration.

The relationship of the intercept, $n$, to the virus concentration is only incidental depending upon the relative concentrations of virus and toxin in a particular preparation. Further, the manner in which the toxic activity varies with concentration of toxin is at present unknown.

In one series of experiments the concentration of $V$ has been varied while the parameter, $t$, was held constant. The relationship of the virus concentration to cellular destruction is illustrated in the following representative experiments.

Relationship of Viral Dose and Cellular Response. In separate experiments replicate cultures were prepared for infection and exposed to various concentrations of virus for a period of two hours. In these experiments the range of virus concentration was from $0.83 \times 10^{6}$ to $60 \times 10^{6} \mathrm{PFU}$ per culture. After the period of exposure, the cultures were washed thoroughly (five times with balanced salt solution) to remove residual inoculum and then incubated 24 hours with immune serum. By the usual procedure described in previous sections the fraction of cells destroyed was estimated. In Fig. 6 the resulting data are recorded; the $\log C_{0} / C$ is plotted against the virus concentration. In the concentration range studied the cellular response is an exponential function of the virus concentration. However, it will be noted that upon extrapolation to zero, there is an apparent intercept indicating some heterogeneity of the system. 
Fate of Virus in the Initiation of Infection. If, in the system under investigation, a multiplicity of infection can occur and if a Poissor distribution is operative, then it should be possible to predict from the number of cells reacting the number of virus particles that have reacted at any given time.

To test this hypothesis the disappearance of free virus from the system was measured. A HeLa cell monolayer was incubated overnight in maintenance solution containing $10 \%$ equine serum, washed four times. with balanced salt solution. At zero time and at 15 minute intervals for 90 minutes, $0.05 \mathrm{ml}$ samples were withdrawn, immediately diluted

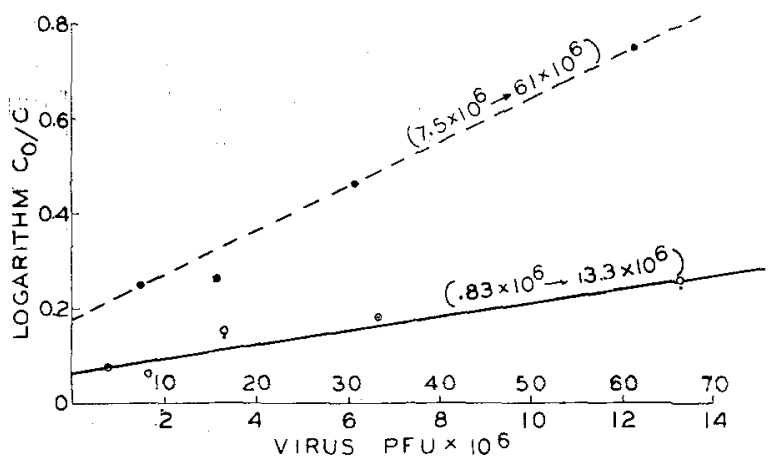

Fig. 6. Relation of Virus Concentration to Cell Destruction

The open circles $(O)$, closed circles $(O)$, and dots (u) represent data from three separate experiments. The same pool of virus was used in the two experiments resulting in the lower curve; another pool of virus was used to obtain the upper curve.

In all instances, each point represents a single culture which was exposed to the indicated amount of virus for two hours. After washing five times to remove excess virus the cultures were :supplemented with antiviral sermm and the surviving fraction of cells was determined at 24 hours.

and plated for determination of their viral content. During the period of incubation at $37^{\circ} \mathrm{C}$, the culture was gently shaken to insure adequate mixing and uniform exposure of the cellular monolayer. As a control, an empty culture bottle was similarly washed, inoculated, incubated and sampled. Within the limits of error of the plaque assay procedure, there was no change in the virus concentration of such a control in the periods studied (up to three hours).

The results of a representative experiment are shown in Fig. 7. In this instance the multiplicity of exposure was three. It is seen that there is a linear relationship between the logarithm of the concentration of virus and time. A similar linear relationship is evident when $\ln \left(V_{0} / V\right)$ is ploted against time and may be expressed by the equation:

$$
\ln \left(V_{o} / V_{t}\right)=k t
$$


where $V_{o}$ and $V_{t}$ are respectively the virus concentration at zero time and any given time $t ; k$ is a reaction constant which under the above eircumstances is $0.146 \mathrm{~min} .^{-1}$.

Thus the loss of virus from the medium follows first order kinetics, the rate of disappearance of virus at any time being dependent on the instantaneous concentration of free virus. Similar observations have

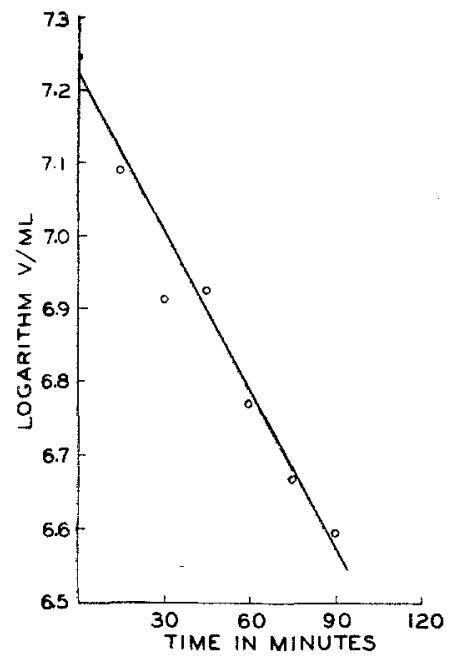

Fig. 7. Disappearance from Medium of Poliovirus Type 1 (Mahoney) on Monolayers of HeLa Cells

A monolayer culture of HeLa cells was exposed to $4 \mathrm{ml}$ of virus suspension containing $1.76 \times 10^{7} \mathrm{PWU} / \mathrm{mI}$ (multiplicity of exposure $=3 \mathrm{PFU} / \mathrm{cell}$ ). During the period of exposure the culture was incubated at, $37^{\circ} \mathrm{O}$ and gently shaken. At 15 -minute intervals, $0.05 \mathrm{ml}$ samples of the medium were withdrawn, immediately diluted and plated for their viral content. than $15 \%$ of the virus disappearing can be accounted for on the basis of cells infected.

This relatively rapid rate of disappearance of virus from the medium might reflect the formation of an early unstable cell-virus complex. It is possible, however, to recover only about $5 \%$ of the virus which has disappeared from the medium by repeatedly washing the monolayer. An additional $0.1 \%$ of the "lost" virus is detectable by lysing the cells with $0.05 \mathrm{M}$ sodium citrate in $0.1 \mathrm{M}$ sodium chloride.

The observation that in a cell suspension there are 20 times as many infected cells (determined by plating cells for plaque forming units) as 
there are infectious particles after the cells are lysed offers evidence of an eclipse phase, but the amount of virus utilized in this manner is relatively small.

The assumption that all the cells in the system have an equal adsorbing capacity is very likely not true. Thus virus particles on the cells would not be distributed in strict accordance with a Poisson distribution. The HeLa cell line used in the experiments described has not been clonally purified and we have actually isolated cell populations that differ from the wild line in these early reactions with poliovirus. However, it should be noted that Dulbecco has shown that in the bacteria-bacteriaphage system only very large differences of the surface of the cells affect the calculated multiplicity ${ }^{6}$. Thus the possibility remains that at high multiplicities of exposure there may be associated with HeLa cells a phenomenon of viral inactivation other than that attributable to the process of infection.

\section{Discussion}

In several respects the present findings are reminescent of data obtained previously with influenza virus?. Initiation of infection proceeds in twe stages distinguishable by sensitivity to viral immüne serum. Recently other workers applied this same serum technique to the study of Newcastle disease virus with essentially the same findings 8 .

In each case antibody prepared against the virus can react to prevent further integration of a preformed cell-virus complex. These observations as well as others with bacteria-phage are pertinent to the consideration of the basis of the effect of neutralizing antibody ${ }^{9}$. The simple interpretation that the geometry of the virus is altered by its combination with antibody so as to prevent, by steric hindrance, reaction with the cell seems inadequate.

When the interaction of cell and virus is followed by the cell counting technique, the kinetics obtained indicate the rate of the first reaction depends upon both the concentration of virus and susceptible cells. In the presence of high concentrations of virus, the reaction behaves as a pseudo-first order reaction. This would obtain whether one were dealing with an interaction of free virus and cells or if the reaction was the transition of a transient unstable complex to a more stable one, i. e., stable to dilution. The further development of the infection with the acquisition of serum-resistance is clearly a first-order reaction whose rate depends upon the instantaneous concentration of the stable cellvirus complex.

There is a discrepancy between the number of plaques which can be induced in a monolayer of cells by a viral preparation at high dilution and the number of cells the same preparation will infect at high 
concentration. This holds if one allows for inefficiency caused by multiplicity of infection and for the toxic effect. In all these experiments the concentration of virus expressed must be considered a minimal value, since the number of plaques detectable depends upon the sensitivity of the cells used for assay. Even in the best preparations using sensitive human amnion cells, thirty particles as visualized in the electron microscope are necessary to produce one plaque ${ }^{10}$. This could mean that even at very low concentrations, a large fraction of the virus reacts with cells and is inactivated without initiating viral production or death of cells. While there are other interpretations, the phenomenon may be related to the present data obtained at high concentrations of virus. The loss of viral activity in the presence of HeLa cells or kidney cells which cannot be accounted for by the eclipse phase of the infection must be considered further.

The aberration in the kinetic curve of Reaction I which was described for some lines of virus seems entirely attributable to the presence of a toxic material. This activity may be the residual effect of the previous sequence of infection from which the viral inoculum was prepared, in which case the two periods of cytopathic activity described may represent one mechanism of cellular disintegration. The nature of this toxic effect will be considered in detail elsewhere.

It is now feasible with the development of these technique to attempt a quantitative analysis of viral pathogenicity and cellular resistance in terms of these early discrete stages of host-virus interaction.

\section{Summary}

1. A method has been developed for the quantitation of cellular injury in tissue cultures of HeLa cells.

2. Crude viral preparations obtained from infected tissue cultures were found to induce, upon transfer to fresh cultures of cells, two distinct periods of cytopathogenic activity. One of these occurs early; the other follows the usual period of viral development. Only preparations of certain lines of poliovirus produce the rapid effect.

3. An analysis of the second period of cytopathogenic activity showed the potential for cytopathology (which is expressed at six to seven hours after infection) is established early in the interaction of cell and virus.

4. Two phases of this interaction have been recognized. One is the establishment of a cell-virus complex which is stable to dilution and sensitive to the neutralizing effect of antiviral immune serum. Its rate is dependent upon the concentration of both cells and virus. The loss of viral activity from the inoculum which accompanies the reaction was found to follow first-order kinetics. This loss is in excess of that calculated to be necessary to establish the degree of infection observed. 
5. The second phase of interaction is the conversion of the stable cell-virus complex to one which is resistant to the action of immune serum. The conversion proceeds by first-order kinetics. The half-life of the reacting complex is approximately 30 minutes.

\section{Bibliography}

${ }^{1}$ Wenner, H. A., C. A. Miller, P. Kamitsuka, and J.C. Wilson: Preparation and standardization of antiserums prepared with the three known types of poliomyelitis virus. Amer. J. Hyg. 59, 221 (1954). — ${ }^{2}$ Eagle, H.: The specific amino acid requirements of a human carcinoma cell (strain HeLá) in tissue culture. J. exper. Med. (Am.) 102, 37 (1955). $-{ }^{3}$ Scherer, W. F.: The utilization of a pure strain of mammalian cells (Earle) for the cultivation of viruses in vitro. I. Multiplication of pseudorabies and herpes simplex viruses. Amer. J. Path. 29, 113 (1953). - ${ }^{4}$ Dulbecco, R., and M. Vogt: Plaque formation and isolation of pure lines with poliomyelitis viruses. J. exper. Med. (Am.) 97, 167 (1954). - ${ }^{5}$ Fogh, J.: Relation between multiplicity of exposure, adsorption and cytopathogenic effect of poliomyelitis virus in monkey kidney tissue culture. Virology 1, 324 (1955). - ${ }^{6}$ Dulbecco, $R .:$ On the reliability of the Poisson Distribution as a distribution of the number of phage particles infecting individual bacteria in a population. Genetics (Am.) 34, 122 (1949). - ${ }^{7}$ Ishida, N., and W.W. Ackermann: Growth characteristics of influenza virus. Properties of the initial cell-virus complex. J. exper. Med. (Am.) 104, 501 (1956). - ${ }^{8}$ Rubin, H., and R. M. Franklin: personal communication. - ${ }^{9}$ Nagano, $Y$., and $M$. Mutai: Etudes sérologiques sưr le bactériophage; adsorption du bactériophage neutralisé sur la bactérie hôte. C. r. Soc. Biol. 148, 766 (1954). — ${ }^{10}$ Schwerdt, C. E., and F. L. Schaffer: Purification of poliomyelitisvirus propagated in tissue culture. Virology $\mathbf{2}$ 665 (1956). 